\title{
Téoros
}

Revue de recherche en tourisme

\section{Les vacances des nouveaux retraités}

Les idées préconçues sont bouleversées

\section{Jean Stafford et Marcel Samson}

Volume 4, numéro 2, juillet 1985

Les clientèles touristiques : un tour d'horizon

URI : https://id.erudit.org/iderudit/1080628ar

DOI : https://doi.org/10.7202/1080628ar

Aller au sommaire du numéro

Éditeur(s)

Université du Québec à Montréal

ISSN

0712-8657 (imprimé)

1923-2705 (numérique)

Découvrir la revue

Citer cet article

Stafford, J. \& Samson, M. (1985). Les vacances des nouveaux retraités : les idées préconçues sont bouleversées. Téoros, 4(2), 23-36.

https://doi.org/10.7202/1080628ar d'utilisation que vous pouvez consulter en ligne.

https://apropos.erudit.org/fr/usagers/politique-dutilisation/ 


\title{
Les idées préconçues sont bouleversées
}

\section{Les vacances des nouveaux retraités}

\author{
par Jean Stafford et Marce/ Samson*
}

Est-ce que les comportements de vacances des personnes âgés se transforment après la retraite? Quels sont les impacts de l'arrêt de travail sur les comportements de vacances des personnes âgées? Ce sont lầ des questions qui sont au coeur d'une recherche ${ }^{11}$ qui a comme objectif d'observer l'effet de la retraite sur les départs en vacances des personnes qui ont acquis récemment le statut de retraité(e) ${ }^{(2)}$. Dans le présent article cependant, nous insisterons surtout sur les pratiques actuelles de vacances des nouveaux retraités constitués par la cohorte d'âge des 66-71 ans.

\section{Le contexte de l'étude}

Dans le cadre de cette recherche, une upersonne retraitéen est une personne âgée qui touche actuellement la pension de sécurité de la vieillesse du Canada. Outre son utilité méthodologique, cette définition nous permet de respecter le statut de retraitées des personnes qui niont pas effectué de travail rémunéré avant leur retraite. Par ailleurs, pour des raisons théoriques et methodologiques, nous avons choisi d'interviewer la population-cible des 66-71 ans. Les effets directs de la retraite sont encore ressentis par ce groupe d'áge; la période de vie étudiée avant la retraite s'étalant sur les cinq années précédentes, du mëme coup les personnes qui avaient pris leur retraite avant 65 ans étaient incluses.

Au plan géographique, les 502 individus échantillonnés résident dans la ville centrale, c'est-â-dire la ville de Montréal de mème que les municipalités dont le territoire est presque totalement enclavé dạs les limites de cette demière (Outremont, Westmount, Montréal-Nord, Anjou, StLéonard, Montréal-Est, Montréal-Ouestl.

Les entrevues ont toutes été réalisées au domicile même de chacun des répondants. Ces entrevues ont été conduites entre le 1er novembre et le 22 décembre 1984 par des étudiantes et des étudiants du Module de gestion et d'intervention

"Jesan Stafford est professeur au département d'études urbaines de I'U.Q.A.M. at Marcel Samson est professeur cherchour à I'I.N.R.S.-Urbanisation. touristique de I'Université du Québec à Montréal ${ }^{(3)}$. Compte tenu du nombre de questionnaires complétés, du nombre de personnes initialement sélectionnées et des personnes qui se sont averées inéligibles, le taux de réponse s'établit à $50,7 \%$.

\section{Des retraités qui partent...}

De I'analyse de l'ensemble de l'échantillon, il se dégage que la mobilité de la cohorte d'ãge des 66-71 ans est plus forte que nous aurions pu prévoir. Les quelques études parues sur les comportements touristiques des Québécois et des Canadiens révèlent que les personnes âgées de plus de 65 ans présentent un taux de départ généralement inférieur à la moyenne de la population adulte. Ainsi d'après une enquête sur les comportements des Canadiens menée en 1984, $44 \%$ des personnes âgées de 65 ans et plus font un voyage de vacances (déplacement d'au moins quatre nuits) au cours $d^{\prime} u n e$ période de douze mois ${ }^{|4|}$.

L'étude Samson-Barnier de 1980 indiquait un taux de départ de $55 \%$ pour les 65 ans et plus dans la région métropolitaine de recensement de Montréal alors que celle de Laplante-Nadeau (1983) indiquait un taux de $51 \%$ pour la région économique de Montréal(5). Or dans le tableau 1 cijoint, on observe que les taux de depart avant et après la retraite atteignent au moins $70 \%$.

Etonnants, ces chiffres le deviennent moins lorsque nous nous rappelons que l'enquête portait sur les individus de la cohorte 66-71 ans et non sur l'ensemble de la population des 65 ans et plus. En outre, ce sont des urbains dont la résidence est située dans la ville centrale telle que définie plus haut. Or, toutes les études le confirment, la population urbaine a une propension à voyager beaucoup plus grande que la population rurale.

\section{Des citoyens qui ne sont pas parmi les plus riches...}

Le concept de départ en vacances a toujours été plus ou moins lié à la richesse relative des individus. Autrement dit, s'il faut du temps libre pour partir, il faut aussi pouvoir se le permettre... Ainsi, il n'est pas surprenant que $41 \%$ des individus avant leur retraite et $37 \%$ après leur retraite avancent des raisons financières comme cause de non-départ. Si cela con= stitue un empechement majeur, quoique bien d'autres motifs sont aussi avancés, il reste que les départs en vacances sont une pratique de consommation à laquelle on semble renoncer difficilement.

Le tableau 2 reflète bien la distribution inégale de la richesse à l'intérieur de notre cohorte.

On observe que $67.5 \%$ de l'ensemble de l'échantillon a un revenu familial de moins de 15000 dollars. Par ailleurs, plus du tiers de l'échantillon est composé d'individus vivant seuls dont $60,5 \%$ ont un revenu inférieur à 10000 dollars. Ce sont

Tableau 1

Taux de départ en vacances avant et après la retraite pour les 66-71 ans, en pourcentage
Cinq dernière années
avant la retraite
Après la retraite

$\begin{array}{llll}\text { Tres souvent/souvent } & 70 \% & \text { Au moins une fois } & 75 \% \\ \text { Jamais } & 30 \% & \text { Jamais } & 25 \%\end{array}$




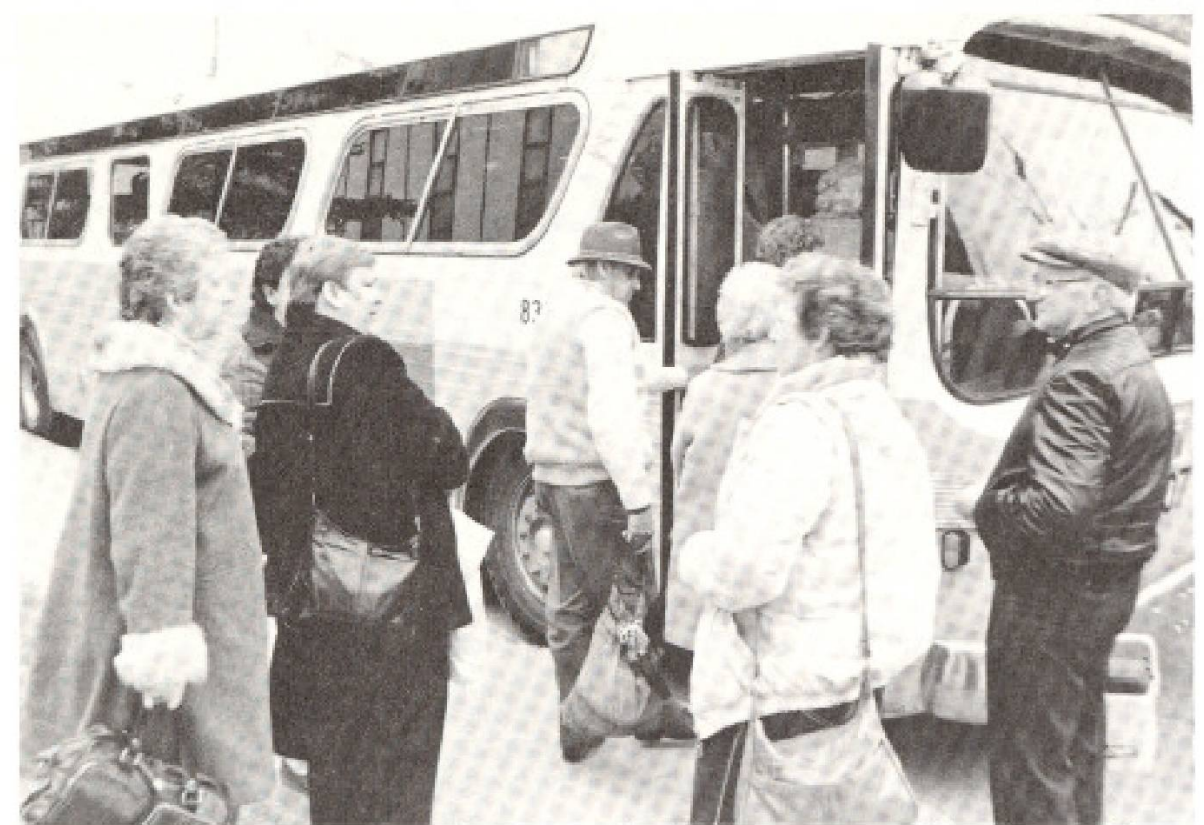

Les départs en vacances sont une pratique de consommation à laquelle on semble renoncer difficilement. sen tirent le mieux compte tenu des différents paiements de transfert qui leur mềme cohorte d'âge, ce taux ne dépasse pas $50 \%$. Enfin, la possession d'une automobile étant un facteur important dans le départ en vacances, $91 \%$ des femmes vivant seules n'en posseddent pas alors que ce taux tombe a $57 \%$ pour les hommes vivant seuls et a $36 \%$ chez les couples. les individus vivant avec un conjoint qui sont versés.

II n'est pas sans intérêt de préciser que $79 \%$ des individus wivant seuls sont des femmes lcélibataires, veuves ou divorcéesl dont la majorité n'avaient pas d'emploi rémunéré avant la retraite. $64 \%$ d'entre elles ont un revenu annuel de moins de 10000 dollars. Comme pour les hommes seuls, elles sont locataires de leur logement dans une proportion de $70 \%$ alors que pour les couples de cette

\section{Stabilité du comportement de vacances}

La retraite, nous le savons, peut entrainer une foule de ruptures au plan économique et social; l'ancien mode de vie liế au travail pour certains, le rite de passage que constitue la réception du paiement de sécurité de la vieillesse pour d'autres peut entraîner des changements importants dans les rapports avec la famille et l'entourage. Ainsi en est-il des vacances où un nombre limité de comportements sont possibles ${ }^{(6)}$.

Le premier type de comportement face aux vacances en est un de continuité. Ce sont les vacanciers stables qui ont, à peu de choses près, les meimes comportements de vacances avant et aprés la retraite, $62,7 \%$ des nouveaux retraités sont des vacanciers stables (vacances ici est toujours entendu au sens de depart en vacances).

Tableau 2

Revenu familial pour la cohorte 66-71 ans par catégorie et selon la structure des ménages, en pourcentage

\begin{tabular}{cccc}
\hline $\begin{array}{c}\text { Ensemble de } \\
\text { I'échantillon (100\%) }\end{array}$ & $\begin{array}{c}\text { Individus } \\
\text { seuls }(36,2 \%)\end{array}$ & $\begin{array}{c}\text { Individus } \\
\text { mariés }(63,9 \%)\end{array}$ \\
\hline $0-9999$ & 33,4 & 60,5 & 18,0 \\
$10000-14999$ & 34,1 & 20,3 & 41,8 \\
$15000-19999$ & 15,9 & 11,4 & 18,4 \\
$20000-24999$ & 6,5 & 3,8 & 7,9 \\
$25000-29999$ & 3,5 & 1,3 & 4,6 \\
30000 et plus & 6,7 & 2,5 & 9,0 \\
\hline
\end{tabular}

Le deuxième type de comportement regroupe ceux qui ont renoncé, pour une raison ou une autre, à avoir des vacances. $7,2 \%$ des nouveaux retraités sont des exvacanciers.

Le troisième type de comportement est composé de ceux qui accedent aux vacances en même temps qu'à la retraite. $12,7 \%$ des nouveaux retraites sont des néo-vacanciers.

Le quatrième type de comportement face aux vacances en est un de continuite négative. Ce sont ceux qui ne prenaient pas de vacances avant la retraite et qui r'en prennent pas après la retraite. $17,3 \%$ des nouveaux retraites sont des nonvacanciers.

Ces derniers chiffres montrent clairement que pour une majorité de retraites, les comportements de vacances avant et après la retraite sont marqués par la stabilité. En fait, $20 \%$ seulement des individus de notre échantillon adoptent un comportement différent après leur retraite : ce sont les ex-vacanciers et les néo-vacanciers. Des études menées dans les années 70 , en France particulièrement, avaient amené leurs auteurs à constater cette même stabilité de comportement parmi les nouveaux retraites ${ }^{[7]}$.

Pour les fins du présent article, nous situerons maintenant les principales modalitês de vacances de cette clientèle touristique de nouveaux retraités.

\section{Quelles destinations de vacances?}

Parmi les retraitếs qui partent en vacances, une proportion de $50 \%$ disent retourner au même endroit année aprês année.

On ne sera pas surpris de constater que les Etats-Unis, avec $28,5 \%$ au total, exercent un attrait aussi important que la grande région montréalaise, II est particulièrement remarquable que l'État de la Floride à lui seul accueille $19 \%$ de ces vacanciers ( $14 \%$ environ de l'ensemble des nouveaux retraités). Cette situation doit être interprétée dans le contexte des changements socio-culturels du comportement vacancier survenus depuis le début des années 60. A la recherche d'un climat moins rigoureux, plusieurs retraités québécois émigrent maintenant vers la Floride durant la saison d'hiver. La croissance de la mobilité gênérale des retraités, l'augmentation du niveau des rentes de retraite Irentes de l'Etat, pensions d'entreprise,...) et, plus recemment, l'abaissement de l'age de la retraite pour certains sont des facteurs qui ont contribué à l'ampleur du phénomène. Cette migration annuelle a ainsi favorisé l'émergence d'un milieu de villégiature qui exerce un attrait important chez les Québécois francophones. 
Ceux qui restent au Québec se comportent sensiblement comme l'ensemble des vacanciers montréalais. La région de Montréal ou plus précisément les régions touristiques limitrophes que sont les Laurentides-Lanaudiere et l'Estrie ressortent clairement sur toutes les autres destinations du Quêbec.

\section{Les modes d'hébergement}

La propension des nouveaux retraités à sortir du Québec pour leurs vacances se reflète dans les modes d'hébergement : $41 \%$ des vacanciers se logent dans les hôtels ou motels. On est surpris de constater que les séjours à la résidence principale des parents ou amis n'atteignent que $23 \%$; on se serait attendu à un plus fort pourcentage étant donné les origines provinciales de plusieurs Montréalais et du coût moins élevé de ce type de sêjour. Par ailleurs, ce dernier pourcentage augmente lorsquion y ajoute les $4,5 \%$ de personnes qui affirment passer habituellement leurs vacances à la résidence secondaire de parents ou amis. Au troisieme rang, la résidence secondaire possédée ou louée ou encore appartenant à des parents ou amis atteint $14,3 \%$. Enfin, $15 \%$ des nouveaux retraités utilisent généralement deux modes d'hébergement durant leurs vacances et cela se répartit entre les types de sêjour ci-haut mentionnés.
Les hébergements de type social ne sont pratiquement pas utilisés et cela apparaît comme une carence importante chez cette catégorie de vacanciers pour qui les coûts financiers d'un sejour ne doivent pas être prohibitifs. II faut comprendre que ce type d'hébergement est fortement méconnu par cette clientele; cela est aussi un indice supplêmentaire de la stabilité du comportement de vacances dans les premières années de la retraite.

\section{La période de vacances et la durée du séjour}

Un autre aspect de cette relative stabilité du comportement se retrouve dans la periode choisie pour partir en vacances. La plupart des retraités prennent leurs vacances à la même période que la majorité des vacanciers québécois : $52,4 \%$ choisissent les mois de juillet-aoüt. Pourquoi en serait-il autrement? Ce sont lâ les mois les plus agréables au plan climatique pour ceux qui restent à l'intérieur des frontières.

II est clair que le nombre de retraités qui prennent des vacances à l'extérieur, dans le sud des États-Unis particulièrement, se reflètent dans le pourcentage non négligeable $(27,7 \%)$ de ceux qui êtalent leurs séjours sur deux périodes. Outre juillet-

Tableau 3

Destination des retraités fidèles au mème endroit de vacances, en pourcentage

\begin{tabular}{lrcc}
\hline \multicolumn{1}{c}{ Destinations } & $\%$ & Total Québec & Total Canada \\
\hline Région de Montréal & 28,5 & & \\
Région de Québec & 8,6 & & \\
Gaspésie & 7,5 & & \\
Lac St-Jean & 4,3 & $51,6 \%$ & \\
Ailleurs au Québec & 2,7 & & \\
Ontario & 5,9 & & \\
Provinces maritimes & 2,7 & & \\
Ailleurs au Canada & 3,2 & \\
& & & \\
Nord des États-Unis & 9,7 & & \\
Sud des Etats-Unis & 18,8 & \\
Antilles-Mexique & 1,6 & \\
Europe & 5,9 & \\
Reste du monde & 0,5 & \\
\hline
\end{tabular}

Tableau 4

Périodes de l'année et durée des séjours des nouveaux retraités, en pourcentage

\begin{tabular}{lrlr}
\hline Périodes & $\%$ & Durée des séjours & $\%$ \\
\hline Décembre - Mars & 9,8 & $4-7$ jours & 19,8 \\
Avril - Juin & 4,5 & 8 -14 jours & 16,4 \\
Juillet - Août & 52,4 & $15-21$ jours & 22,2 \\
Septembre - Novembre & 5,6 & Plus de 21 jours & 41,7 \\
Deux périodes & 27,7 & & \\
\hline
\end{tabular}

août, cet autre moment fort des vacances est vraisemblablement concentré sur les mois de décembre-mars oủ on retrouve déjá un contingent de $9,8 \%$ de vacanciers.

Si $20 \%$ des personnes aggées font de courts sêjours qui ne dépassent pas 4-7 jours, un groupe de $42 \%$ partent à l'extérieur de leur domicile pour une durée de plus de 21 jours. C'est un trait important du comportement vacancier des retraités. Non limités en principe par le facteur temps, ces derniers prolongent leur séjour au-delá des limites imposées par les contraintes du travail rémunéré ou des obligations familiales plus nombreuses des ménages qui n'ont pas encore atteint l'étape de la retraite.

\section{La fréquence des départs et les modes de transport utilisés}

Ce que nous venons de signaler à propos de la durée des séjours se répercute aussi dans le nombre de départs annuels de vacances. Press de $40 \%$ des vacanciers partent deux fois ou plus et $44,6 \%$ partent au moins une fois l'an. Ces chiffres révelent une mobilité beaucoup plus grande que chez les non-retraités et jettent quelques doutes sur le fait que la diminution des ressources financieres a cet agge de la vie constitue un facteur important de changement dans la pratique des vacances.

$46 \%$ de notre cohorte affirment posséder une automobile, ce taux correspond exactement au nombre de ceux qui utilisent ce moyen de transport pour partir en vacances. L'avion vient au deuxième rang avec $22 \%$ et s'explique en bonne partie par les séjours dans l'État de la Floride. Mis a part l'avion, le transport en commun le plus utilise est l'autobus avec $12 \%$ : quant au train, a $3 \%$, aussi bien dire que c'est un mode de transport complètement délaissé par cette clientèle. Enfin, 17\% des voyageurs utilisent deux modes de transport, avion et automobile le plus fréquemment.

\section{Que cherche-t-on à travers ces vacances?}

C'est dans une proportion de $29 \%$ que le principal but de vacances est la visite de parents ou d'amis, ce qui correspond à la proportion de personnes dont le mode d'hébergement en sẹjour de vacances est la résidence principale ou secondaire de ces même parents ou amis. La visite de lieux touristiques reconnus $(23 \%)$ et la migration vers des régions au climat plus accueillant $(20 \%)$ complètent les objectifs de vacances des Montréalais âgés. Invités a préciser, les répondants ont confirmé a plus de $50 \%$ que la visite des parents et amis constitue un critère important dans la décision de partir en vacances; c'est suite a la page 36 
U.S. TRAVEL DATA CENTER, National Travel Survey (trimestriel). Washington. D.C. 1974

\section{Clientèle internationale}

CANADA, Statistique Canada, Voyages entre le Canada et les autres pays [catalogue 66-001, trimestriell, Ottawa, 1931

CANADA, Tourisme Canada, Attitudes des Allemands de la République fédérale allemande envers le Canada comme destina tion touristique, Ottawa, 1984, $13 \mathrm{p}$.

CANADA, Tourisme Canada, Attitude des Hollandais à l'endroit du Canada comme destination de vacances, Ottawa, 1984.

CANADA, Tourisme Canada, Attitude des résidents du Royaume-Uni à l'endroit du Canada comme destination de voyages. Ottawa, 1983

CANADA, Tourisme Canada, Attitude des Suisses à l'endroit du Canada comme des. tination de vacances, Ottawa, 1984.

GARONER-SMITH, Gillian, Europe begins to add up, Tourism Management, vol. $5, \mathrm{n}^{\circ} 2$. (June 1984), pp. 158-160

HILL HODEL, George, The Japan air Travel Market in TTRA 12th annual conference proceedings (1981), U.S., University of Utah, 1981, pp, 273-284.

O'CONNOR, Thomas, Marketing to the International Visitor, The Cornell Hotel and Restaurant Quarterly, vol. 21, $n^{\text {" }} 4$ (February 19811. pp. 53-59.

ORGANISATION DE COOPERATION ET DE DEVELOPPEMENT ECONOMIOUES (OCDE). Politique du Tourisme et tourisme interna tional dans les pays membres de l'OCDE (annuel), Paris, 1967.

ORGANISATION MONDIALE DU TOURIS. $M E$, La fréquentation des principales destinations touristiques 1979-80, Madrid, 1981 $329 \mathrm{p}$.

ORGANISATION MONDIALE DU TOURIS

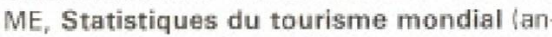
nuelj. Madrid, 1947

SCHMIDHAUSER, Hanspeter, The Swiss Travel Market and its Role within the Main Tounst Generating Countries of Europe, Revue de Tourisme, $n^{\circ} 4$ (1976), pp. 15-18.

THIFFAULT, GUY, Le tourisme international au Québec en provenance des Ėtats-Unis et des autres pays 1973-1980. Québec, MICT, 1983, $63 \mathrm{p}$.

\section{Clientèles spéciales}

\section{- personnes âgées}

CENTRE INTERNATIONAL DE GERONTOLOGIE SOCIALE, Colloque Tourisme et 3ème âge, Paris, 1973, $110 \mathrm{p}$.

CRISSEY, Yvonne, Over forty nine the active affluent, in TTRA 11th conference proceedings: (1980). U.S., University of Utah, 1980, pp. $127+134$

DONFUT, Claudine, Vacances : loisir du $3 \mathrm{e}$ àge. France, Cagnes-sur-Mer, CNRO, 1972, $193 \mathrm{p}$.

NESTLEBAUM, Karen, Meetings for Seniors, Meetings \& Conventions, January 1985 pp, 93-99,
SHOEMAKER, Storne, Marketing to older travelers, The Cornell Hotel \& Restaurant Administration quartely, Vol. $25, \pi^{\circ} 2$, (august 1984), pp. 84-91.

TONGREN, Hale $N_{\text {. }}$ Travel Plans of the over-65 market Pre and Postretirement, Journal of Travel Research, vol, XIX, $n^{\circ} 2$ (tall 1980), pp. 7-11.

- Autres

BLANK, Uel, The motropolitan area tourist : is comprehensive analysis, in TTRA 10th conference proceedings, U.S., University of Utah, 1979, pp. 105-112.

BURKART, A.J., Trends in UK business travel overseas, Tourism Management, vol. $4, \mathrm{n}^{\circ} 1$ (march 1983), pp. 54.57.

BUSH, Melinda, Capturing Business Travelers: the travelagency connection. The Cornell Hotel and Restaurant Administration Quarterly, vol. 23, $\mathrm{n}^{\circ}$ 2, (august 1982). pp. 58-61.

DISCOVER AMERICA NATIONAL CONFE RENCE (1978), Emerging Travel Markets the Woman Traveler, Washington D.C. Travel Institute, $1978,30 \mathrm{p}$.

GlBBONS, Rioy, Singles and Couples, in TTRA 11th conference proceedings, pp. 113-126.

HENRY, Regina, A case history; the wornan business traveler and Western international Hoteds, in TTRA 11th conference proceedings, pp. 105-112.

\section{suite de la page 25}

aussi un moyen d'evacuer le quotidien et une excellente occasion de connaitre de nouvelles régions.

En terminant, force est de constater que la cohorte des 66-71 ans, ces nouveaux retraités, ont un comportement de vacances qui bouleverse les idées préconcues qu'on pouvait encore entretenir à leur sujet. C'est, on l'a observé, une catégorie dont le taux et la fréquence des départs en vacances sont relativement élevés et qui n'a rien à envier sous cet aspect à la population des non-retraités.

II faut souligner que ces personnes avaient $25-30$ ans au sortir de la deuxième guerre mondiale. C'est donc une génération qui, â l'àge adulte, est entrée de plein pied dans cette période de l'histoire qu devait connaître un essor sans précédent de I'industrie touristique. Contrairement aux générations précédentes pour qui les départs en vacances étaient une conduite en bonne partie réservée à la bourgeoisie, les vacances de masse sont apparues timidement pour devenir un fait de société au cours des années soixante. II ne faut pas ignorer que le $1 / 4$ des nouveaux retraités, composé surtout de femmes seules, $n^{\prime}$ ont pas accès aux vacances. Cela correspond grosso modo à la proportion des nondéparts parmi la population de la région métropolitaine.
MARKET PROBE INTERNATIONAL INC., A report on the year-round honeymoon travel market 1974. New York: Market Probe, 1974,54 p.

MATTHEWS, Louise et LUCAS-GIRARDVILLE, Jean-Marie, Le tourisme a la portée de l'enfant, Québec, MICT, 1980, $106 \mathrm{p}$.

MENARD, Johanne et al. L'intégration potentielle touristique des personnes handicapées: étude de marché sur les besoins et les attentes des personnes handicapées physiques du Québec face au tourisme. Montréal, Kéroul Inc., 1984

MEUNIER, Gilles, YOUNG, Linda, Ėtude sur les motivations, les attentes et les habitudes des familles à faible revenu en regard des activités de loisir touristique. Québec: MLCP, 1983, 72 p.

SOUCY, Louiselle, SIMARD, Brigitte, BEAUDRY, Jocelyn, Le profil de l'étudiant voyageur, Montréal : OTEO, 1984, 25 p.

Woman Business Travelers: satisfying the Needs of a Growing New Market. The Cornell Hotel \& Restaurant Administration Quartely, vol. 19, n० 4. (February 1979), pp. 67-69.

* Daniet Dumas est bibliothecaire au Centre d'études du tourisme. Tous les documents cités dans cêtte bibliographie sont disponibles pour consultation ou prèt au Centre d'études du tourisme, 1420, Saint-Denis, à Montréal. (514) 282-9613.

Finalement cette généralisation des conduites de vacances au lendemain de la retraite constitue, au-delá de toutes les ruptures, un point de raccord avec le passé immédiat et sans doute un facteur important d'insertion sociale. $f$

\section{Références}

1) Certé recherche a été subventionnée par le Con seil des recherches en sciences humaines du Canada (CRSHC).

(2) Les vacances sont antendues ici comme un départ d'au moins 4 jours consdcutifs hors du domićile habituel et pour des fins de loisir.

(3) M. Daniel Bbesu, etudiant stagiaire a l'INRS, a coordonné les travail des intervieweurs sur le terrain et a participe a ranalyse,

(4) Enquête menée par le Print Measurement Bureau et citce dans Tourime Canada, wol. 5, no 9. novembre 1984

(5) SAMSON M. et V. BAFNIER, Vacances at Tourisme 80 , Montréal, INRS-Urbanisation. Etudes et Documents no, 30. 1982; Laplante, Marc, Gens qui partent of gens qui restent UQAM. Département d'Etudes urbaines. Docu ment no 1. 1983.

(6) L'analyse de ces différents comportements saront au coeur d'un rapport qui sera publié cette anné par les auteurs du present afticle.

(7) CRIBIEF, F, les vocances a heure de la revate conduites diage, conduites de generation, Loisir et Societe, vol, 2, no 2, P.U, 0. 1979- DONFUT, C., Vacances: Loisir du 3e sge, Paris, Géron. tologie (numéro spécial), no 20,1972 